\title{
Ground waves in atomic chains with bi-monomial double-well potential
}

\author{
Michael Herrmann*
}

March 8, 2022

\begin{abstract}
Ground waves in atomic chains are traveling waves that corresponds to minimal non-trivial critical values of the underlying action functional. In this paper we study FPU-type chains with bi-monomial double-well potential and prove the existence of both periodic and solitary ground waves. To this end we minimize the action on the Nehari manifold and show that periodic ground waves converge to solitary ones. Finally, we compute ground waves numerically by a suitable discretization of a constrained gradient flow.
\end{abstract}

Keywords: $\quad$ Fermi-Pasta-Ulam chain with double-well potential, Nehari manifold, ground waves

MSC (2010): 37K60, 47J30, 74J30

\section{Contents}

1 Introduction

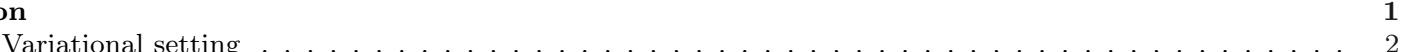

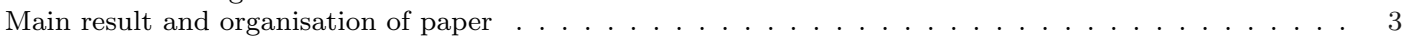

Comparison with other results $\ldots \ldots \ldots \ldots \ldots \ldots \ldots \ldots$

2 Preliminaries and Nehari manifold 5

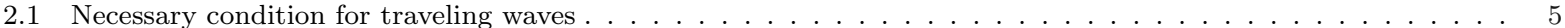

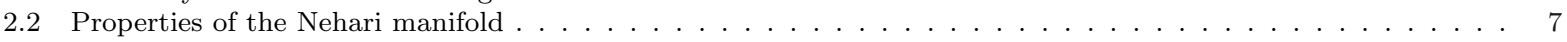

3 Ground waves as Nehari minimizers of the action 9

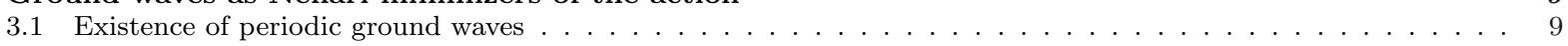

3.2 Convergence to solitary ground waves $\ldots \ldots \ldots \ldots \ldots \ldots \ldots \ldots$

4 Numerical solutions

\section{Introduction}

Atomic chains with nearest neighbor interactions, which are usually called FPU-type chains, are ubiquitous in physics and materials science as they provide simple atomistic models for solids. They are moreover prototypical examples for nonlinear Hamiltonian lattice equations and shed light on the dynamical properties of discrete media with dispersion.

The lattice equation for infinite FPU-type chains stems from Newton's law of motion and reads

$$
\ddot{x}_{j}=\Phi^{\prime}\left(x_{j+1}-x_{j}\right)-\Phi^{\prime}\left(x_{j}-x_{j-1}\right),
$$

where $\Phi$ is the interaction potential and $x_{j}=x_{j}(t) \in \mathbb{R}$ denotes the position of atom $j \in \mathbb{Z}$ at time $t$.

*Universität des Saarlandes, FR Mathematik, michael.herrmann@math.uni-sb.de 
Coherent structures such as traveling waves are of particular interest in the analysis of nonlinear lattice equations since they can be regarded as the nonlinear fundamental modes and describe how energy propagates through the chain. Traveling waves are special solutions to (1) which depend on a one-dimensional phase variable $\varphi=j-\sigma t$ via the ansatz

$$
x_{j}(t)=r j+v t+X(j-\sigma t) .
$$

Here, $\sigma$ the phase speed, $r$ and $v$ are given constants, and the profile function $X$ satisfies

$$
\sigma^{2} X^{\prime \prime}(\varphi)=\Phi^{\prime}(r+X(\varphi+1)-X(\varphi))-\Phi^{\prime}(r+X(\varphi)-X(\varphi-1)) .
$$

It can easily be shown that this advance-delay differential equation is equivalent to the nonlinear eigenvalue problem

$$
\sigma^{2} W=\mathcal{A} \Phi^{\prime}(\mathcal{A} W)+\mu
$$

for the function $W$ defined by $W(\varphi)=r+X^{\prime}(\varphi)$. Here, $\mu$ is some constant of integration and the convolution operator $\mathcal{A}$ is defined by

$$
(\mathcal{A} W)(\varphi)=\int_{\varphi-1 / 2}^{\varphi+1 / 2} W(s) \mathrm{d} s .
$$

Traveling waves in atomic chains have been studied intensively during the last two decades, and the existence of several types of solutions to (2) has been established for various interaction potentials by different methods. For small amplitude waves, the standard references are [FP99] for the continuum approximation of near-sonic waves and [Ioo00] for bifurcation results via spatial dynamics. The existence of waves with non-small amplitudes has been proven by different variational or critical point techniques, see for instance [FW94, FV99, PP00, SW97, FM02, SZ07, HR10], which we discuss below in greater detail.

In this paper we consider chains with double-well potential, which play an important role in the atomistic theory of martensitic phase transitions. In order to keep the presentation as simple as possible, we restrict ourselves to bi-monomial potentials with

$$
\Phi(w)=d_{p}|w|^{p}-d_{q}|w|^{q} \quad \text { with } \quad 2<p, \quad 1<q<p,
$$

and by a simple scaling we can achieve that $d_{p}=d_{q}=1$. We further restrict our considerations to the case $\mu=0$, see the discussion below, and seek non-trivial solutions to the traveling wave equation

$$
\sigma^{2} W+\mathcal{A} \Psi_{q}(\mathcal{A} W)=\mathcal{A} \Psi_{p}(\mathcal{A} W)
$$

where the functions $\Psi_{r}$ are defined by $\Psi_{r}(w):=r \operatorname{sgn}(w)|w|^{r-1}$.

Variational setting Due to the Hamiltonian nature and the shift invariance of (1), there is a variational characterization of traveling waves with prescribed $\sigma^{2}$. To see this for both periodic and solitary waves, we introduce a positive parameter $K \in(0, \infty]$. For finite $K$, we study $2 K$ periodic waves and regard $I_{K}:=(-K, K]$ as the periodicity cell; the case $K=\infty$ corresponds to solitary waves defined on $I_{\infty}:=\mathbb{R}$. In what follows we denote by $\mathrm{L}^{r}\left(I_{K}\right)$ and $\mathrm{W}^{1, r}\left(I_{K}\right)$ the usual Sobolev spaces of $I_{K}$-periodic functions (or functions on $\mathbb{R}$ for $K=\infty$ ), and write

$$
\|\cdot\|_{r, I_{K}} \quad \text { and } \quad\langle\cdot, \cdot\rangle_{I_{K}}
$$

for the norm on $\mathrm{L}^{r}\left(I_{K}\right)$ and the scalar product in $\mathrm{L}^{2}\left(I_{K}\right)$, respectively. Moreover, we define

$$
\mathrm{X}_{K}:=\left\{W \in \mathrm{L}^{2}\left(I_{K}\right): \mathcal{A} W \in \mathrm{L}^{q}\left(I_{K}\right) \cap \mathrm{L}^{p}\left(I_{K}\right)\right\}
$$


as ansatz space for periodic and solitary waves.

The variational formulation of (6) relies on the Lagrangian action functional

$$
\mathcal{L}_{K}(W):=\frac{1}{2} \sigma^{2}\|W\|_{2, I_{K}}^{2}+\mathcal{Q}_{K}(W)-\mathcal{P}_{K}(W),
$$

which is well defined on $\mathrm{X}_{K}$, where $\frac{1}{2} \sigma^{2}\|W\|_{2, I_{K}}^{2}$ can be regarded as the kinetic energy, and

$$
\mathcal{Q}_{K}(W):=\int_{I_{K}}|\mathcal{A} W|^{q} \mathrm{~d} \varphi, \quad \mathcal{P}_{K}(W):=\int_{I_{K}}|\mathcal{A} W|^{p} \mathrm{~d} \varphi
$$

give the two contributions to the potential energy $\mathcal{P}_{K}-\mathcal{Q}_{K}$. The functionals $\mathcal{L}_{K}, \mathcal{Q}_{K}$, and $\mathcal{P}_{K}$ are also Gâteaux-differentiable $\mathrm{X}_{K}$ with derivatives

$$
\begin{aligned}
\partial \mathcal{L}_{K}(W) & =\sigma^{2} W+\partial \mathcal{Q}_{K}(W)-\partial \mathcal{P}_{K}(W) \\
\partial \mathcal{Q}_{K}(W) & =\mathcal{A} \Psi_{q}(\mathcal{A} W) \\
\partial \mathcal{P}_{K}(W) & =\mathcal{A} \Psi_{p}(\mathcal{A} W)
\end{aligned}
$$

and we conclude that each solution $W \in \mathrm{X}_{K}$ to the traveling wave equation (6) is a critical point of $\mathcal{L}_{K}$, and vice versa.

Main result and organisation of paper In this paper we establish the existence of periodic and solitary ground waves for the special potential (5). By definition, a ground wave is a traveling wave that corresponds to a minimal non-trivial critical value of the action functional $\mathcal{L}_{K}$. Our main result can be summarized as follows.

Theorem 1. Let $\sigma^{2}>0$ be given. Then, for each $K \in(0, \infty]$ there exists a ground wave $W_{K} \in \mathrm{X}_{K}$ which satisfies (6) as well as

$$
0<\mathcal{L}_{K}\left(W_{K}\right)=\min \left\{\mathcal{L}_{K}(W): W \in \mathrm{X}_{K} \backslash\{0\} \text { with } \partial \mathcal{L}_{K}(W)=0\right\}
$$

and which is non-constant provided that $K$ is sufficiently large. Moreover, solitary ground waves can be approximated (in some strong sense) by period ground waves.

Before commenting on the main ideas in the proof, we proceed with the following remarks:

1. Our proof reveals that each ground waves $W_{K}$ satisfies

$$
\mathcal{L}_{K}\left(W_{K}\right)=\min _{W \in \mathrm{X}_{K} \backslash\{0\}} \max _{\zeta>0} \mathcal{L}_{K}(\zeta W)
$$

that means ground waves are not minimizers but saddle points of $\mathcal{L}_{K}$.

2. The traveling wave equation (6) and the properties of the convolution operator $\mathcal{A}$, see Lemma 2 below, ensure that travelling waves belong to $\mathrm{BC}^{1}(\mathbb{R})$ and that solitary ground waves are homoclinic via $\lim _{\varphi \rightarrow \pm \infty} W(\varphi)=0$. Moreover, each ground wave is supersonic in the sense of $\sigma^{2}>0 \geq \Phi^{\prime \prime}(0)$.

3. The set of all ground waves for given $\sigma^{2}>0$ is obviously invariant under shifts $W(\varphi) \rightsquigarrow$ $W\left(\varphi+\varphi_{0}\right)$, reflections $W(\varphi) \rightsquigarrow W(-\varphi)$, and sign changes $W(\varphi) \rightsquigarrow-W(\varphi)$.

4. Since $\Phi$ is a double-well potential, there exists an amplitude threshold, this means a lower bound for the strain amplitude $\|A W\|_{\infty, I_{K}}$ independent of $\sigma$ and $K$, see Remark 8 below. This is different from the case of convex potentials as these support near sonic waves with arbitrarily small amplitudes [FP99, Her10]. 
5. Numerical simulations as presented in $\S 4$ indicate that the solitary ground waves provided by Theorem 1 have, for sufficiently small $\sigma$, oscillatory tails. This is also different from the case of convex potentials, where the profile function $W$ of solitary waves usually has only one local extremum [Her10].

6. Although our existence result is restricted to bi-monomial double-well potentials, we expect that the underlying ideas can - for the price of more technical effort - be generalized to more general double-well potentials $\Phi(w)=-\Phi_{1}(w)+\Phi_{2}(w)$ as long as $(i)$ the partial potentials $\Phi_{1}$ and $\Phi_{2}$ are convex, $(i i) \Phi_{1}$ dominates $\Phi_{2}$ for all sufficiently small $|w|$, and (iii) $\Phi_{2}$ grows superquadratically and, at least for large $|w|$, faster than $\Phi_{1}$.

We also mention that $\mathcal{L}_{K}$ has, at least for $K<\infty$, infinitely many critical points in $\mathrm{X}_{K}$. In particular, each ground wave for $K / n$ with $n \in \mathbb{N}$ is also a critical point of $\mathcal{L}_{K}$, but qualitatively different types of traveling waves might exist as well. Moreover, for $K=\infty$ we expect that there also exist traveling waves with non-decaying profile $W$. Of particular importance are phase transitions waves, which are heteroclinic connections of two period waves corresponding to either one of wells. Unfortunately, very little is known about their existence. The only available results concern bi-quadratic potentials, which allow for solving (2) by Fourier methods [TV05, SZ09], or almost bi-quadratic potentials, for which we can employ perturbation methods [HMSZ12]. It remains a challenging task to find alternative, maybe variational, existence proofs for phase transition waves that apply to more general double-well potentials.

In order to prove Theorem 1 , we introduce in $\S 2$ the Nehari manifold $\mathrm{M}_{K}$, which has co-dimension 1 and contains all non-trivial critical points of $\mathcal{L}_{K}$. In $\S 3.1$ we employ the direct method from the calculus of variations and show that the functional $\mathcal{L}_{K}$ attains its minimum on $\mathrm{M}_{K}$ for $K<\infty$. Afterwards in $\S 3.2$ we demonstrate that periodic ground waves with $K \rightarrow \infty$ provide minimizing sequences for $\mathcal{L}_{\infty} \mid \mathrm{M}_{\infty}$ and converge to a solitary ground wave. Finally, we compute ground waves numerically in $\S 4$.

Comparison with other results We finally compare our results and methods with previous work on traveling waves in FPU-type chains.

At first we mention that the amplitude threshold implies that traveling waves in double-well potentials cannot be obtained by perturbation arguments applied to the trivial wave $W(\varphi) \equiv 0$. Moreover, also the available existence proofs via constrained optimization does not cover potential (5). Specifically, both the method used by Friesecke and Wattis in [FW94] (minimization of kinetic energy under prescribed potential energy) and the approach discussed in [Her10] (maximization of potential energy under prescribed kinetic energy) require - among other conditions - that the asymptotic state of the solitary wave $w_{*}=\lim _{\varphi \rightarrow \pm} W(\varphi)$ is a minimum of the tilted potential

$$
\Phi_{*}(w)=\Phi\left(w_{*}+w\right)-\Phi^{\prime}\left(w_{*}\right) w-\Phi\left(w_{*}\right),
$$

and this condition is apparently not satisfied for the waves provided by Theorem 1 (notice that in our case we have $w_{*}=0$ and hence $\left.\Phi_{*}=\Phi\right)$.

Theorem 1 is, however, not the first existence result for traveling waves in bi-monomial doublewell potentials. Smets and Willem [SW97] combine the Mountain Pass Theorem with weak convergence methods to establish the existence of solitary waves for a large class of potentials including (5). However, these waves are not necessarily ground waves and it is not clear whether they can be approximated by periodic ones. A further drawback is that solutions provided by mountain pass arguments are hard to compute numerically.

Closely related to our work is the discussion of ground waves given by Pankov in [Pan05, Section 3.4]. The results presented there imply the assertions of Theorem 1 for the special case 
$q=2$ and are likewise based on the Nehari manifold and approximation with periodic waves. The proof, however, is different as it employs the Mountain Pass Theorem and the Palais-Smale condition for $K<\infty$; see $\S 3$ for more details.

A variant of the Mountain Pass Theorem was also used by Schwetlick and Zimmer [SZ07] to construct homoclinic waves for certain double-well potentials. These waves satisfy $W=w_{*}+\tilde{W}$ with $\lim _{|\varphi| \rightarrow \infty} \tilde{W}(\varphi)=0$, where $w_{*}$ is one of the local minimizer of $\Phi$. The key idea is that the relative profile $\tilde{W}$ can be regarded as solitary wave with respect to the tilted potential (7), which has, at least for certain double-well potentials, sufficiently nice properties. The relation to our approach becomes apparent in the periodic case. Instead of tilting the potential we can impose the constraint $\left|I_{K}\right|^{-1} \int_{I_{K}} W \mathrm{~d} \varphi=w_{*}$, and we easily check that critical points of $\mathcal{L}_{K}$ now satisfy the traveling wave equation (3) with Lagrangian multiplier $\mu \in \mathbb{R}$. Due to the constraint, however, the corresponding traveling waves are not ground waves for the action $\mathcal{L}_{K}$.

\section{Preliminaries and Nehari manifold}

In this section we develop our variational framework for both finite and infinite $K$ and introduce the Nehari manifold, on which we minimize the action in $\S 3$. The parameter $\sigma^{2}>0$ is from now on fixed.

We first summarize some properties of the convolution operator $\mathcal{A}$. In particular, we show that $\mathcal{A}$ maps $\mathrm{L}^{2}\left(I_{K}\right)$ compactly into $\mathrm{L}^{r}\left(I_{K}\right)$ provided that $1 \leq r<\infty$ and $K<\infty$.

Lemma 2. Let $K \in(0, \infty]$ and $1 \leq r<\infty$ be given. Then, the linear operator $\mathcal{A}$ maps $\mathrm{L}^{r}\left(I_{K}\right)$ continuously into $\mathrm{W}^{1, r}\left(I_{K}\right) \subset \mathrm{BC}(\mathbb{R})$ with

$$
\left\|(\mathcal{A} W)^{\prime}\right\|_{r, I_{K}} \leq 2\|W\|_{r, I_{K}}, \quad\|\mathcal{A} W\|_{\infty, I_{K}} \leq\|W\|_{r, I_{K}}, \quad\|\mathcal{A} W\|_{r, I_{K}} \leq\|W\|_{r, I_{K}} .
$$

Moreover, $W_{n} \rightarrow W_{\infty}$ weakly in $\mathrm{L}^{2}\left(I_{K}\right)$ implies $\mathcal{A} W_{n} \rightarrow \mathcal{A} W_{\infty}$ pointwise for all $K$ and also strongly in $\mathrm{L}^{r}\left(I_{K}\right)$ for $K<\infty$.

Proof. Thanks to $(\mathcal{A} W)^{\prime}(\varphi)=W(\varphi+1 / 2)-W(\varphi-1 / 2)$ and since Hölder's inequality implies

$$
|(\mathcal{A} W)(\varphi)|^{r} \leq \int_{\varphi-1 / 2}^{\varphi+1 / 2}|W(s)|^{r} \mathrm{~d} s
$$

all estimates follows immediately. Moreover, the pointwise convergence $\mathcal{A} W_{n} \rightarrow \mathcal{A} W$ follows from the definition of $\mathcal{A}$ in (4), and implies the strong convergence for $K<\infty$ due to $\mathrm{L}^{\infty}\left(I_{K}\right) \subset \mathrm{L}^{r}\left(I_{K}\right)$ and the Dominated Convergence Theorem.

Remark 3. For $K=\infty$ or $K \notin \pi \mathbb{Q}$, the operator $\mathcal{A}: \mathrm{L}^{2}\left(I_{K}\right) \rightarrow \mathrm{L}^{2}\left(I_{K}\right)$ has only trivial kernel.

Proof. The operator $\mathcal{A}$ diagonalizes in Fourier space via $\mathcal{A} \mathrm{e}^{\mathrm{i} k \varphi}=\varrho(k / 2) \mathrm{e}^{\mathrm{i} k \varphi}$ for all $k \in \mathbb{R}$, where $\varrho(\kappa)=\sin (\kappa) / \kappa$, and the assertion follows immediately.

Notice that Lemma 2 implies $\mathrm{X}_{K}=\mathrm{L}^{2}\left(I_{K}\right)$ for $K<\infty$, as well as $\mathcal{P}_{K}(W) \leq\|W\|_{2, I_{K}}^{p-q} \mathcal{Q}_{K}(W)$ for all $K \in(0, \infty]$ and $W \in \mathrm{X}_{K}$.

\subsection{Necessary condition for traveling waves}

A key property of the action functional $\mathcal{L}_{K}$ is that its restriction to the positive ray $\zeta>0 \mapsto \zeta W$ has a unique maximizer for every non-degenerate $W$. To see this, we start with an auxiliary result about the maximizers of certain tri-monomial functions. 
Lemma 4. For any $c=\left(c_{2}, c_{q}, c_{p}\right) \in \mathbb{R}_{+}^{3}$ let the function $\lambda_{c}: \mathbb{R}_{+} \rightarrow \mathbb{R}$ be defined by

$$
\lambda_{c}(\xi):=c_{2} \xi^{2}+c_{q} \xi^{q}-c_{p} \xi^{p} .
$$

Then, the function $\bar{\xi} \mathbb{R}_{+}^{3} \rightarrow \mathbb{R}$ with

$$
\bar{\xi}(c):=\operatorname{argmax}_{\xi>0} \lambda_{c}(\xi)
$$

is well-defined, continuous, and positive.

Proof. The function $f_{c}$ with

$$
f_{c}(\xi):=\xi \lambda_{c}^{\prime}(\xi)=2 c_{2} \xi^{2}+q c_{q} \xi^{q}-p c_{p} \xi^{p}
$$

is continuous on $R_{+}$and satisfies

$$
f_{c}(\xi)>0 \quad \text { and } \quad f_{c}(\xi)<0
$$

for small and large $\xi$, respectively. Therefore, there exists at least one zero $\bar{\xi}>0$ with

$$
0=f_{c}(\bar{\xi})=2 c_{2} \bar{\xi}^{2}+q c_{q} \bar{\xi}^{q}-p c_{p} \bar{\xi}^{p} .
$$

Thanks to (8) we also find

$$
\bar{\xi} f_{c}^{\prime}(\bar{\xi})=(4-2 p) c_{2} \bar{\xi}^{2}+\left(q^{2}-q p\right) c_{q} \bar{\xi}^{q}<0,
$$

for any such zero $\bar{\xi}$, and this implies that $f_{c}$ has precisely one zero $\bar{\xi}=\bar{\xi}(c)$. In particular, we have

$$
\lambda_{c}^{\prime}(\xi)>0 \quad \text { and } \quad \lambda_{c}^{\prime}(\xi)<0
$$

for $\xi<\bar{\xi}(c)$ and $\xi>\bar{\xi}(c)$, respectively, and we conclude that $\bar{\xi}(c)$ is a global maximizer of $\lambda_{c}$ with

$$
0<\lambda_{c}(\bar{\xi}(c))=\max _{\xi>0} \lambda_{c}(\xi)
$$

Finally, using (8) once more we verify the estimates

$$
\max \left\{\left(\frac{2 c_{2}}{p c_{p}}\right)^{1 /(p-2)},\left(\frac{q c_{q}}{p c_{p}}\right)^{1 /(p-q)}\right\} \leq \bar{\xi}(c) \leq \max \left\{\left(\frac{4 c_{2}}{p c_{p}}\right)^{1 /(p-2)},\left(\frac{2 q c_{q}}{p c_{p}}\right)^{1 /(p-q)}\right\},
$$

which in turn imply the claimed continuity of $\bar{\xi}$ since we have $\lambda_{c_{n}} \rightarrow \lambda_{c}$ uniformly as $c_{n} \rightarrow c \in \mathbb{R}_{+}^{3}$ on each compact subset of $\mathbb{R}_{+}$.

Corollary 5. For $K \in(0, \infty]$ and $W \in \mathrm{X}_{K}$ with $\mathcal{A} W \neq 0$ let

$$
\bar{\zeta}_{K}(W):=\bar{\xi}\left(\frac{1}{2} \sigma^{2}\|W\|_{2, I_{K}}^{2}, \mathcal{Q}_{K}(W), \mathcal{P}_{K}(W)\right)
$$

Then, we have

$$
0<\mathcal{L}_{K}\left(\bar{\zeta}_{K}(W) W\right)=\max _{\zeta>0} \mathcal{L}_{K}(\zeta W)
$$

and $W_{n} \rightarrow W$ strongly in $\mathrm{L}^{2}\left(I_{K}\right)$ with $\mathcal{A} W \neq 0$ implies $\bar{\zeta}_{K}\left(W_{n}\right) \rightarrow \bar{\zeta}_{K}(W)$. 
In view of Corollary 5 , we introduce the functional

$$
\mathcal{F}_{K}(W):=\left.\frac{\mathrm{d}}{\mathrm{d} \zeta} \mathcal{L}_{K}(\zeta W)\right|_{\zeta=1}=\left\langle\partial \mathcal{L}_{K}(W), W\right\rangle_{I_{K}}=\sigma^{2}\|W\|_{2, I_{K}}^{2}+q \mathcal{Q}_{K}(W)-p \mathcal{P}_{K}(W),
$$

which is well defined and Gâteaux differentiable on $\mathrm{L}^{2}\left(I_{K}\right)$ with

$$
\partial \mathcal{F}_{K}(W)=2 \sigma^{2} W+q \mathcal{A} \Psi_{q}(\mathcal{A} W)-p \mathcal{A} \Psi_{p}(\mathcal{A} W) .
$$

We further define the Nehari manifold by

$$
\mathrm{M}_{K}:=\left\{W \in \mathrm{X}_{K}: W \neq 0, \quad \mathcal{F}_{K}(W)=0\right\},
$$

and notice that $\bar{\zeta}_{K}(W) W \in \mathrm{M}_{K}$ for all $W$ with $\mathcal{A} W \neq 0$. Moreover, $W \in \mathrm{M}_{K}$ implies $\mathcal{A} W \neq 0$, and for $\mathcal{A} W \neq 0$ we have

$$
W \in \mathrm{M}_{K} \Longleftrightarrow \bar{\zeta}_{K}(W)=1 \quad \Longleftrightarrow \quad \mathcal{L}_{K}(W)=\max _{\zeta>0} \mathcal{L}_{K}(\zeta W) .
$$

Remark 6. Each non-vanishing traveling wave $W \in \mathrm{X}_{K}$ belongs to $\mathrm{M}_{K}$ and $\mathrm{BC}^{1}(\mathbb{R})$.

Proof. $W \neq 0$ combined with the traveling wave equation (6) implies $\mathcal{A} W \neq 0$ thanks to $\sigma \neq 0$, and $W \in \mathrm{M}_{K}$ follows since testing (6) with $W$ gives $\mathcal{F}_{K}(W)=0$. Moreover, $W \in \mathrm{BC}^{1}(\mathbb{R})$ is a direct consequence of Lemma 2 and (6).

Our strategy for proving the existence of ground waves is to show that $\mathcal{L}_{K}$ attains its minimum on $\mathrm{M}_{K}$. We can then conclude that each minimizer satisfies the traveling wave equation (6), and Remark 6 guarantees that the minimum is in fact the smallest non-trivial critical value of $\mathcal{L}_{K}$.

\subsection{Properties of the Nehari manifold}

We next derive some estimates on the Nehari manifold.

Lemma 7. There exist positive constants $c$ and $C$, which both are independent of $K$ but can depend on $\sigma$, such that

1. $c \leq\|W\|_{2, I_{K}}^{2} \leq C \mathcal{L}_{K}(W)$,

2. $c \leq\|\mathcal{A} W\|_{\infty, I_{K}}^{2} \leq C \mathcal{L}_{K}(W)$,

3. $c \leq \mathcal{L}_{K}(W)$,

4. $c \leq \mathcal{P}_{K}(W) \leq C \mathcal{L}_{K}(W)$,

5. $\mathcal{Q}_{K}(W) \leq C \mathcal{L}_{K}(W)$,

6. $\left\langle\partial F_{K}(W), W\right\rangle_{I_{K}} \leq-c$,

hold for all $W \in \mathrm{M}_{K}$.

Proof. Employing $\mathcal{F}_{K}(W)=0$ and Lemma 2 we estimate

$$
q \mathcal{Q}_{K}(W) \leq \sigma^{2}\|W\|_{2, I_{K}}^{2}+q \mathcal{Q}_{K}(W)=p \mathcal{P}_{K}(W) \leq p\|\mathcal{A} W\|_{\infty, I_{K}}^{p-q} \mathcal{Q}_{K}(W),
$$

where $W \in \mathrm{M}_{K}$ implies $A W \neq 0$ and hence $\mathcal{Q}_{K}(W)>0$. Therefore, and thanks to Lemma 2, we find

$$
\|W\|_{2, I_{K}} \geq\|\mathcal{A} W\|_{\infty, I_{K}} \geq\left(\frac{q}{p}\right)^{1 /(p-q)}
$$


and this implies

$$
p \mathcal{P}_{K}(W)=\sigma^{2}\|W\|_{2, I_{K}}^{2}+q \mathcal{Q}_{K}(W) \geq \sigma^{2}\|W\|_{2, I_{K}}^{2} \geq \sigma^{2}\left(\frac{q}{p}\right)^{2 /(p-q)} .
$$

Due to $\mathcal{F}_{K}(W)=0$ we also have

$$
\mathcal{L}_{K}(W)=\sigma^{2}\left(\frac{1}{2}-\frac{1}{p}\right)\|W\|_{2, I_{K}}^{2}+\left(1-\frac{q}{p}\right) \mathcal{Q}_{K}(W) \geq \sigma^{2}\left(\frac{1}{2}-\frac{1}{p}\right)\|W\|_{2, I_{K}}^{2},
$$

and combining this estimate with $(9),(10)$, and $\mathcal{F}_{K}(W)=0$ we arrive at the first five assertions. Moreover, a direct computation yields

$$
\begin{aligned}
\left\langle\partial F_{K}(W), W\right\rangle_{I_{K}} & =2 \sigma^{2}\|W\|_{2, I_{K}}^{2}+q^{2} \mathcal{Q}_{K}(W)-p^{2} \mathcal{P}_{K}(W) \\
& =(2-p) \sigma^{2}\|W\|_{2, I_{K}}^{2}+\left(q^{2}-q p\right) \mathcal{Q}_{K}(W) \leq-|p-2| \sigma^{2}\|W\|_{2, I_{K}}^{2},
\end{aligned}
$$

which in turn implies the sixth assertion.

The estimate (9) reveals that the lower bound for the strain amplitude in Lemma 7 is actually independent of $\sigma$, and this implies the amplitude threshold for traveling waves in bi-monomial double-well potentials.

Remark 8. There exists a constant $c$ independent of both $K$ and $\sigma$ such that

$$
\|A W\|_{\infty, I_{K}} \geq c
$$

holds for any non-trivial traveling wave $W$.

The last assertion in Lemma 7 ensures that the Nehari manifold $M_{K}$ is strictly transversal to each positive ray $\zeta>0 \mapsto \zeta W$ with $W \in \mathrm{M}_{K}$. It is therefore clear that minimizers of $\left.\mathcal{L}_{K}\right|_{M_{K}}$ are critical points of $\mathcal{L}_{K}$. Here we give an alternative proof of this assertion that relies on the constrained gradient flow for $\mathcal{L}_{K}$, that is

$$
\frac{\mathrm{d}}{\mathrm{d} \tau} W_{\tau}=-\partial \mathcal{L}_{K}\left(W_{\tau}\right)+\lambda_{K}\left(W_{\tau}\right) \partial F_{K}\left(W_{\tau}\right), \quad \lambda_{K}(W):=\frac{\left\langle\partial \mathcal{L}_{K}(W), \partial \mathcal{F}_{K}(W)\right\rangle_{I_{K}}}{\left\|\partial \mathcal{F}_{K}(W)\right\|_{2, I_{K}}^{2}},
$$

where $\tau$ is the flow time and $\tau \mapsto W_{\tau}$ denotes a curve in $\mathrm{M}_{K}$. This gradient flow is also the starting point for the numerical approximation of ground waves in $\S 4$.

Lemma 9. For each $K \in(0, \infty]$, the initial value problem to the $\mathrm{M}_{K}$-valued $O D E$ (11) is wellposed. Moreover, $\mathcal{L}_{K}$ is strictly decreasing along each non-stationary trajectory and each stationary point solves the traveling wave equation (6).

Proof. Let some initial datum $W_{0} \in \mathrm{M}_{K}$ be given. Lemma 7 implies $\partial \mathcal{F}_{K}\left(W_{0}\right) \neq 0$, and by continuity there exists a small ball $B \subset \mathrm{X}_{K}$ around $W_{0}$ such that the multiplier $\lambda_{K}$ is a well defined and Lipschitz continuous function on $B$. Consequently, there exists a local solution $\tau \in$ $\left[0, \tau_{1}\right) \mapsto W_{\tau} \in \mathbf{X}_{K}$. The definition of $\lambda_{K}$ implies

$$
\frac{\mathrm{d}}{\mathrm{d} \tau} \mathcal{F}_{K}\left(W_{\tau}\right)=\left\langle\partial \mathcal{F}_{K}\left(W_{\tau}\right), \frac{\mathrm{d}}{\mathrm{d} \tau} W_{\tau}\right\rangle_{K}=0,
$$

so $M_{K}$ is indeed invariant under the flow of (11). Moreover, a direct computation gives

$$
\begin{aligned}
\frac{\mathrm{d}}{\mathrm{d} \tau} \mathcal{L}_{K}\left(W_{\tau}\right) & =\left\langle\partial \mathcal{L}_{K}\left(W_{\tau}\right), \frac{\mathrm{d}}{\mathrm{d} \tau} W_{\tau}\right\rangle_{I_{K}} \\
& =\frac{\left\|\partial \mathcal{L}_{K}\left(W_{\tau}\right)\right\|_{2, I_{K}}^{2}\left\|\partial \mathcal{F}_{K}\left(W_{\tau}\right)\right\|_{2, I_{K}}^{2}-\left\langle\partial \mathcal{L}\left(W_{\tau}\right), \partial \mathcal{F}\left(W_{\tau}\right)\right\rangle_{I_{K}}{ }^{2}}{\left\|\partial \mathcal{F}_{K}\left(W_{\tau}\right)\right\|_{2, I_{K}}^{2}} \\
& \geq 0,
\end{aligned}
$$


where the inequality is strict provided that $\partial \mathcal{L}_{K}\left(W_{\tau}\right)$ and $\partial \mathcal{F}_{K}\left(W_{\tau}\right)$ are not co-linear, that means as long as the right hand side in (11) 1 does not vanish. Finally, suppose that $W_{\tau} \equiv W \in \mathrm{M}_{K}$ is stationary under the flow of (11). Then we have

$$
\partial \mathcal{L}_{K}(W)=\lambda_{K}(W) \partial \mathcal{F}_{K}(W),
$$

and testing this identity with $W$ we find

$$
0=\left\langle\partial \mathcal{L}_{K}(W), W\right\rangle_{I_{K}}=\lambda_{K}(W)\left\langle\partial \mathcal{F}_{K}(W), W\right\rangle_{I_{K}}
$$

Lemma 7 now implies $\lambda_{K}(W)=0$, and hence $\partial \mathcal{L}_{K}(W)=0$.

A particular consequence of Lemma 9 is that each minimizer of $\left.\mathcal{L}_{K}\right|_{M_{K}}$ is a stationary point of (11), and thus in fact a traveling wave.

\section{Ground waves as Nehari minimizers of the action}

In this section we finish the proof of Theorem 1 by showing that $\mathcal{L}_{K}$ attains its minimum on the Nehari manifold $\mathrm{M}_{K}$. To this end we employ the direct method for $K<\infty$, and pass afterwards to the limit $K \rightarrow \infty$. For the proofs we define

$$
\ell_{K}:=\inf \mathcal{L}_{K} \mid \mathrm{M}_{K}
$$

and recall that Lemma 7 provides a constant $c>0$ such that $\ell_{K} \geq c$ for all $K \in(0, \infty]$.

\subsection{Existence of periodic ground waves}

We now fix $0<K<\infty$ and employ the compactness of $\mathcal{A}$ to show that each minimizing sequence for $\mathcal{L}_{K} \mid \mathrm{M}_{K}$ contains a subsequence that converges to a minimizer. Alternatively, we could employ critical point techniques as follows. Using similar estimates as in the proof of Lemma 7, one easily shows that the action landscape has a mountain pass geometry via

$$
\mathcal{L}_{K}(0)=0, \quad \inf _{\|W\|_{2, I_{K}}=1} \mathcal{L}_{K}(W) \geq \frac{1}{2} \sigma^{2}, \quad \lim _{\zeta \rightarrow \infty} \mathcal{L}_{K}(\zeta W)=-\infty \quad \text { for } \quad \mathcal{A} W \neq 0,
$$

and the compactness of $\mathcal{A}$ ensures that $\mathcal{L}_{K}$ satisfies the Palais-Smale condition. The existence of non-vanishing critical values is hence implied by the Mountain Pass Theorem, and the PalaisSmale condition guarantees that there is a minimal critical value. The details for this line of argument can, for the special case $q=2$, be found in [Pan05, Section 3.4.1].

Theorem 10. For each $0<K<\infty$ there exists a minimizer of $\mathcal{L}_{K} \mid \mathrm{M}_{K}$.

Proof. Let $\left(W_{n}\right)_{n \in \mathbb{N}} \subset \mathrm{M}_{K}$ be any minimizing sequence for $\left.\mathcal{L}_{K}\right|_{\mathrm{M}_{K}}$. By construction and Lemma 7 , we then have

$$
c \leq\left\|W_{n}\right\|_{2, I_{K}} \leq C, \quad c \leq \mathcal{L}_{K}\left(W_{n}\right) \leq C, \quad c \leq \mathcal{P}_{K}\left(W_{n}\right) \leq C
$$

for some constants $0<c<C<\infty$ independent of $n$. By passing to a (not relabeled) subsequence we can assume that $W_{n} \rightarrow W$ weakly in $\mathrm{L}^{2}\left(I_{K}\right)$, and that $\lim _{n \rightarrow \infty}\left\|W_{n}\right\|_{2, I_{K}}^{2}$ exists, and this implies

$$
\gamma:=\lim _{n \rightarrow \infty}\left\|W_{n}\right\|_{2, I_{K}}-\|W\|_{2, I_{K}} \geq 0 .
$$

Our strategy is now to show that $W$ minimizes $\mathcal{L}_{K}$ on $\mathrm{M}_{K}$. The properties of $\mathcal{A}$, see Lemma 2, guarantee that $\mathcal{A} W_{n} \rightarrow \mathcal{A} W$ pointwise and strongly in $\mathrm{L}^{r}\left(I_{K}\right)$ for all $1<r<\infty$. We therefore 
have $W \in \mathrm{X}_{K}$, and $\mathcal{P}_{K}(W)=\lim _{n \rightarrow \infty} \mathcal{P}_{K}\left(W_{n}\right) \geq c$ yields $\mathcal{A} W \neq 0$ and hence $W \neq 0$. From the definition of $\mathrm{M}_{K}$ we infer that

$$
\begin{aligned}
\ell_{K} & =\mathcal{L}_{K}\left(W_{n}\right) \geq \mathcal{L}_{K}\left(\zeta W_{n}\right) \\
& \geq \frac{1}{2} \zeta^{2} \sigma^{2}\left(\lim _{n \rightarrow \infty}\left\|W_{n}\right\|_{2, I_{K}}^{2}-\|W\|_{2, I_{K}}^{2}\right)+\frac{1}{2} \sigma^{2}\|\zeta W\|_{2, I_{K}}^{2}+\mathcal{Q}_{k}\left(\zeta W_{n}\right)-\mathcal{P}_{K}\left(\zeta W_{n}\right)
\end{aligned}
$$

for all $\zeta>0$, and the above convergencies imply

$$
\mathcal{L}_{K}(\zeta W) \leq \frac{1}{2} \zeta^{2} \sigma^{2} \gamma+\ell_{K}
$$

Choosing $\zeta=\bar{\zeta}_{K}(w) \in(0, \infty)$ gives

$$
\ell_{K} \leq \mathcal{L}_{K}\left(\bar{\zeta}_{K}(W) W\right) \leq \frac{1}{2} \sigma^{2} \bar{\zeta}_{K}(W)^{2} \gamma+\ell_{K},
$$

and we conclude that

$$
\ell_{K}=\max _{\zeta>0} \mathcal{L}_{K}(\zeta W), \quad\|W\|_{2, I_{K}}=\lim _{n \rightarrow \infty}\left\|W_{n}\right\|_{2, I_{K}} .
$$

These identities imply the strong convergence $W_{n} \rightarrow W$ as well as $W \in \mathrm{M}_{K}$ with $\mathcal{L}_{K}(W)=\ell_{K}=$ $\lim _{n \rightarrow \infty} \mathcal{L}_{K}\left(W_{N}\right)$.

To complete our existence proof for periodic ground waves we finally derive an upper bound for $\ell_{K}$ which in turn implies that minimizer of $\mathcal{L}_{K}$ are non-trivial for large $K$.

Lemma 11. We have $\lim \sup _{K \rightarrow \infty} \ell_{K} \leq \ell_{\infty}$.

Proof. Let $W_{\infty} \in \mathrm{M}_{\infty}$ be given. For each $1<K<\infty$ we define $V_{K} \in \mathrm{X}_{\infty}$

$$
V_{K}(\varphi):= \begin{cases}W_{\infty}(\varphi) & \text { for }|\varphi|<K-\frac{1}{2} \\ 0 & \text { else },\end{cases}
$$

and $W_{K} \in \mathrm{X}_{K}$ as the $2 K$-periodic continuation of $\left.V_{K}\right|_{I_{K}}$. This implies

$$
\left\|V_{K}-W_{\infty}\right\|_{2, \mathbb{R}}+\left\|\mathcal{A} V_{K}-\mathcal{A} W_{\infty}\right\|_{r, \mathbb{R}} \stackrel{K \rightarrow \infty}{\longrightarrow} 0,
$$

for all $r \in[1, \infty]$, and by continuity of $\mathcal{L}_{\infty}$ and $\bar{\zeta}_{\infty}$ we get

$$
\mathcal{L}_{\infty}\left(W_{\infty}\right)=\lim _{K \rightarrow \infty} \mathcal{L}_{\infty}\left(V_{K}\right), \quad 1=\bar{\zeta}_{\infty}\left(W_{\infty}\right)=\lim _{K \rightarrow \infty} \bar{\zeta}_{\infty}\left(V_{K}\right) .
$$

Moreover, the properties of $\mathcal{A}$ ensure

$$
\left(\mathcal{A} V_{K}\right)(\varphi)=\left(\mathcal{A} W_{K}\right)(\varphi) \text { for all } \varphi \in I_{K}=(-K, K]
$$

and thus we find

$$
\mathcal{L}_{K}\left(W_{K}\right)=\mathcal{L}_{\infty}\left(V_{K}\right), \quad \bar{\zeta}_{K}\left(W_{K}\right)=\bar{\zeta}_{\infty}\left(V_{K}\right)
$$

Consequently, we have $\mathcal{L}_{\infty}\left(W_{\infty}\right)=\lim _{K \rightarrow \infty} \mathcal{L}_{K}\left(\bar{\zeta}_{K}\left(W_{K}\right) W_{K}\right)$, and $\ell_{K} \leq \mathcal{L}_{K}\left(\bar{\zeta}_{K}\left(W_{K}\right) W_{K}\right)$ yields $\lim \sup _{K \rightarrow \infty} \ell_{K} \leq \mathcal{L}_{\infty}\left(W_{\infty}\right)$. The thesis now follows since $W_{\infty} \in \mathrm{M}_{\infty}$ was arbitrary.

Corollary 12. Let $K<\infty$ be sufficiently large. Then, each minimizer of $\left.\mathcal{L}_{K}\right|_{\mathrm{M}_{K}}$ is non-constant. Proof. The only constant function in $\mathrm{M}_{K}$ is $W(\varphi)=\bar{\zeta}$ with $\bar{\zeta} \in \mathbb{R}$ being the unique maximizer $\bar{\zeta}:=\bar{\xi}\left(\sigma^{2} / 2,1,1\right)>0$ of the function

$$
\xi \mapsto \frac{L_{K}(\xi)}{2 K}=\lambda_{\left(\sigma^{2} / 2,1,1\right)}(\xi)=\frac{1}{2} \sigma^{2} \xi^{2}+\xi^{q}-\xi^{p},
$$

see Lemma 4 and Corollary 5. In particular, for all sufficiently large $K$ we have

$$
\mathcal{L}_{K}(\bar{\zeta})=K \mathcal{L}_{1}(\bar{\zeta})>\ell_{\infty}
$$

and hence $\mathcal{L}_{K}(\bar{\zeta})>\ell_{K}$ due to Lemma 11.

Combing Theorem 10 and Lemma 9 with Remark 6 and Corollary 12 we now obtain our existence result for periodic ground waves as formulated in Theorem 1. 


\subsection{Convergence to solitary ground waves}

Our final goal is to prove that $\mathcal{L}_{\infty}$ attains its minimum on $\mathrm{M}_{\infty}$. Since the operator $\mathcal{A}$ is no longer compact, we cannot argue as in the proof of Theorem 10. Instead, we construct minimizers as limit of period ground waves. The same strategy was used in [Pan05, Section 3.4.2] and some of our key arguments are similar to those presented there.

Theorem 13. $\mathcal{L}_{\infty}$ attains its minimum on $\mathrm{M}_{\infty}$ and we have $\ell_{\infty}=\lim _{K \rightarrow \infty} \ell_{K}$. In particular, each unbounded sequence $\left(K_{m}\right)_{m \in \mathbb{N}}$ has at least one subsequence $\left(K_{n}\right)_{n \in \mathbb{N}}$ with the following property: There exists a corresponding sequence $\left(W_{n}\right)_{n \in \mathbb{N}}$ of period ground waves $W_{n} \in \mathrm{M}_{K_{n}}$ that converges to a solitary ground wave $W_{\infty} \in \mathrm{M}_{\infty}$ in the sense of

$$
\left\|W_{\infty}-V_{n}\right\|_{2, \mathbb{R}} \stackrel{n \rightarrow \infty}{\longrightarrow} 0
$$

where $V_{n} \in \mathrm{X}_{\infty}$ is defined by $V_{n}(\varphi)=W_{n}(\varphi)$ for $\varphi \in I_{K_{n}}$ and $V_{n}(\varphi)=0$ for $\varphi \notin I_{K_{n}}$.

Proof. Step 1: According to Theorem 10 and Remark 6, for each $m$ there exists a periodic traveling wave $\overline{W_{m} \in \mathrm{M}_{K_{m}}}$ which minimizes $\left.\mathcal{L}_{K_{m}}\right|_{\mathrm{M}_{K_{m}}}$. Since $\mathcal{L}_{K}$ and traveling wave equation (6) are invariant under shifts $W \rightsquigarrow W\left(\cdot+\varphi_{0}\right)$, and since the function $\mathcal{A} W_{m}$ is continuous, we can assume that

$$
\left(\mathcal{A} W_{m}\right)(0)=\left\|\mathcal{A} W_{m}\right\|_{\infty, I_{K_{m}}} .
$$

By Lemma 7 and Lemma 11 we also have

$$
c \leq \mathcal{L}_{K_{m}}\left(W_{m}\right) \leq C, \quad c \leq\left\|W_{m}\right\|_{2, I_{K_{m}}} \leq C \quad c \leq\left\|\mathcal{A} W_{m}\right\|_{\infty, I_{K_{m}}} \leq C
$$

and

$$
\mathcal{Q}_{K_{m}}\left(W_{m}\right) \leq C, \quad \mathcal{P}_{K_{m}}\left(W_{m}\right) \leq C
$$

for some constants $0<c<C<\infty$ independent of $m$. Moreover, from (6) we infer, using Lemma 2 and (13), that

$$
\left\|\left(\mathcal{A} W_{m}\right)^{\prime}\right\|_{\infty, I_{K_{m}}} \leq 2\left\|W_{m}\right\|_{\infty, I_{K_{m}}} \leq C\left\|\mathcal{A} W_{m}\right\|_{\infty, I_{K_{m}}} .
$$

In view of (12) we thus obtain

$$
W_{m}(\varphi)=W_{m}(0)+\int_{0}^{\varphi}\left(\mathcal{A} W_{m}\right)^{\prime}(s) \mathrm{d} s \geq d \quad \text { for all }|\varphi| \leq d,
$$

for some constant $d>0$ independent of $m$.

Step 2: By definition, we have $\left\|V_{m}\right\|_{2, \mathbb{R}}=\left\|W_{m}\right\|_{2, I_{K_{m}}} \leq C$ and

$$
\left(\mathcal{A} V_{m}\right)(\varphi)=\left(\mathcal{A} W_{m}\right)(\varphi) \quad \text { for all }|\varphi|<K_{m}-\frac{1}{2} .
$$

We now choose a subsequence $\left(K_{n}\right)_{n \in \mathbb{N}}$ such that $V_{n} \rightarrow W_{\infty} \in \mathrm{L}^{2}(\mathbb{R})$, and Lemma 2 provides the pointwise convergence

$$
\left(\mathcal{A} W_{\infty}\right)(\varphi)=\lim _{n \rightarrow \infty}\left(\mathcal{A} V_{n}\right)(\varphi)=\lim _{n \rightarrow \infty}\left(\mathcal{A} W_{n}\right)(\varphi) .
$$

Moreover, thanks to (6) and (15) we conclude that the sequence $\left(V_{n}\right)_{n \in \mathbb{N}}$ converges pointwise, and combining this with $V_{n} \rightarrow W_{\infty}$ we arrive at the pointwise convergence

$$
W_{\infty}(\varphi)=\lim _{n \rightarrow \infty} V_{n}(\varphi)=\lim _{n \rightarrow \infty} W_{n}(\varphi)
$$

and we conclude that $W_{\infty} \in \mathrm{L}^{2}(\mathbb{R})$ solves the traveling wave equation (6). 
Step 3: For each $0<D<\infty$ and $1 \leq r<\infty$ we have

$$
\int_{-D}^{D}\left|\mathcal{A} W_{\infty}\right|^{r} \mathrm{~d} \varphi=\lim _{n \rightarrow \infty} \int_{-D}^{D}\left|\mathcal{A} W_{n}\right|^{r} \mathrm{~d} \varphi \leq \liminf _{n \rightarrow \infty} \int_{I_{K_{n}}}\left|\mathcal{A} W_{n}\right|^{r} \mathrm{~d} \varphi,
$$

due to the pointwise convergence (16), the estimate (13), and the Dominated Convergence Theorem, and the limit $D \rightarrow \infty$ gives

$$
\mathcal{Q}_{\infty}\left(W_{\infty}\right) \leq \liminf _{n \rightarrow \infty} \mathcal{Q}_{K_{n}}\left(W_{n}\right) \leq C, \quad \mathcal{P}_{\infty}\left(W_{\infty}\right) \leq \liminf _{n \rightarrow \infty} \mathcal{P}_{K_{n}}\left(W_{n}\right) \leq C .
$$

Similarly, using Fatou's Lemma and passing afterwards to $D \rightarrow \infty$ we prove that

$$
\left\|W_{\infty}\right\|_{2, \mathbb{R}}^{2}=\lim _{D \rightarrow \infty} \int_{-D}^{D} W_{\infty}(\varphi)^{2} \mathrm{~d} \varphi \leq \lim _{D \rightarrow \infty} \liminf _{n \rightarrow \infty} \int_{-D}^{D} W_{n}(\varphi)^{2} \mathrm{~d} \varphi \leq \liminf _{n \rightarrow \infty}\left\|W_{n}\right\|_{2, I_{K_{n}}}^{2}
$$

We have now shown that $W_{\infty}$ is a traveling wave, belongs to $\mathrm{X}_{\infty}$, and does not vanish thanks to (14). By Remark 3 and Remark 6 we therefore find $\mathcal{A} W \neq 0$ and hence $W_{\infty} \in \mathrm{M}_{\infty}$.

Step 4: Combining (17) and (18) with $W_{n} \in \mathrm{M}_{K_{n}}$ for all $n \in \mathbb{N} \cup\{\infty\}$ we now estimate

$$
\begin{aligned}
\ell_{\infty} \leq \mathcal{L}_{\infty}\left(W_{\infty}\right) & =\sigma^{2}\left(\frac{1}{2}-\frac{1}{p}\right)\left\|W_{\infty}\right\|_{2, \mathbb{R}}^{2}+\left(1-\frac{q}{p}\right) \mathcal{Q}_{\infty}\left(W_{\infty}\right) \\
& \leq \sigma^{2}\left(\frac{1}{2}-\frac{1}{p}\right) \liminf _{n \rightarrow \infty}\left\|W_{n}\right\|_{2, I_{K_{n}}}^{2}+\left(1-\frac{q}{p}\right) \liminf _{n \rightarrow \infty} \mathcal{Q}_{n}\left(W_{n}\right) \\
& =\liminf _{n \rightarrow \infty} \mathcal{L}_{K_{n}}\left(W_{n}\right)=\liminf _{n \rightarrow \infty} \ell_{K_{n}} .
\end{aligned}
$$

On the other hand, by Lemma 11 we have $\lim \sup _{n \rightarrow \infty} \ell_{K_{n}} \leq \ell_{\infty}$, and thus we find

$$
\ell_{\infty}=\mathcal{L}_{\infty}\left(W_{\infty}\right)=\liminf _{n \rightarrow \infty} \ell_{K_{n}}=\limsup _{n \rightarrow \infty} \ell_{K_{n}}
$$

Consequently, $W_{\infty}$ is in fact a solitary ground wave and we have an equality sign in (19). This implies

$$
\left\|W_{\infty}\right\|_{2, \mathbb{R}}=\lim _{n \rightarrow \infty}\left\|W_{n}\right\|_{2, I_{n}}=\lim _{n \rightarrow \infty}\left\|V_{n}\right\|_{2, \mathbb{R}}
$$

which in turn provides the strong convergence $V_{n} \rightarrow W_{\infty}$. Finally, $\ell_{\infty}=\lim _{K \rightarrow \infty} \ell_{K}$ holds since we have shown that any unbounded sequence $\left(K_{m}\right)_{m \in \mathbb{N}}$ has a subsequence $\left(K_{n}\right)_{n \in \mathbb{N}}$ such that $\ell_{\infty}=\lim _{n \rightarrow \infty} \ell_{K_{n}}$.

\section{Numerical solutions}

In order to illustrate our analytical findings we implemented the following discretization of the constrained gradient flow for $\left.\mathcal{L}_{K}\right|_{\mathrm{M}_{K}}$ :

1. Given $0<K<\infty$, we divide the periodicity cell $(-K, K]$ into $N$ equidistant grid points and approximate all integrals by Riemann sums.

2. We choose a small flow time $\Delta \tau$ and minimize the action on $\mathrm{M}_{K}$ by iterating the following two update steps:

(a) We compute an explicit Euler step for (11), this means we update $W$ tangential to $\mathbf{M}_{K}$ via

$$
W \mapsto W-\triangle \tau\left(\partial \mathcal{L}_{K}(W)-\lambda_{K}(W) \partial \mathcal{F}_{K}(W)\right)
$$



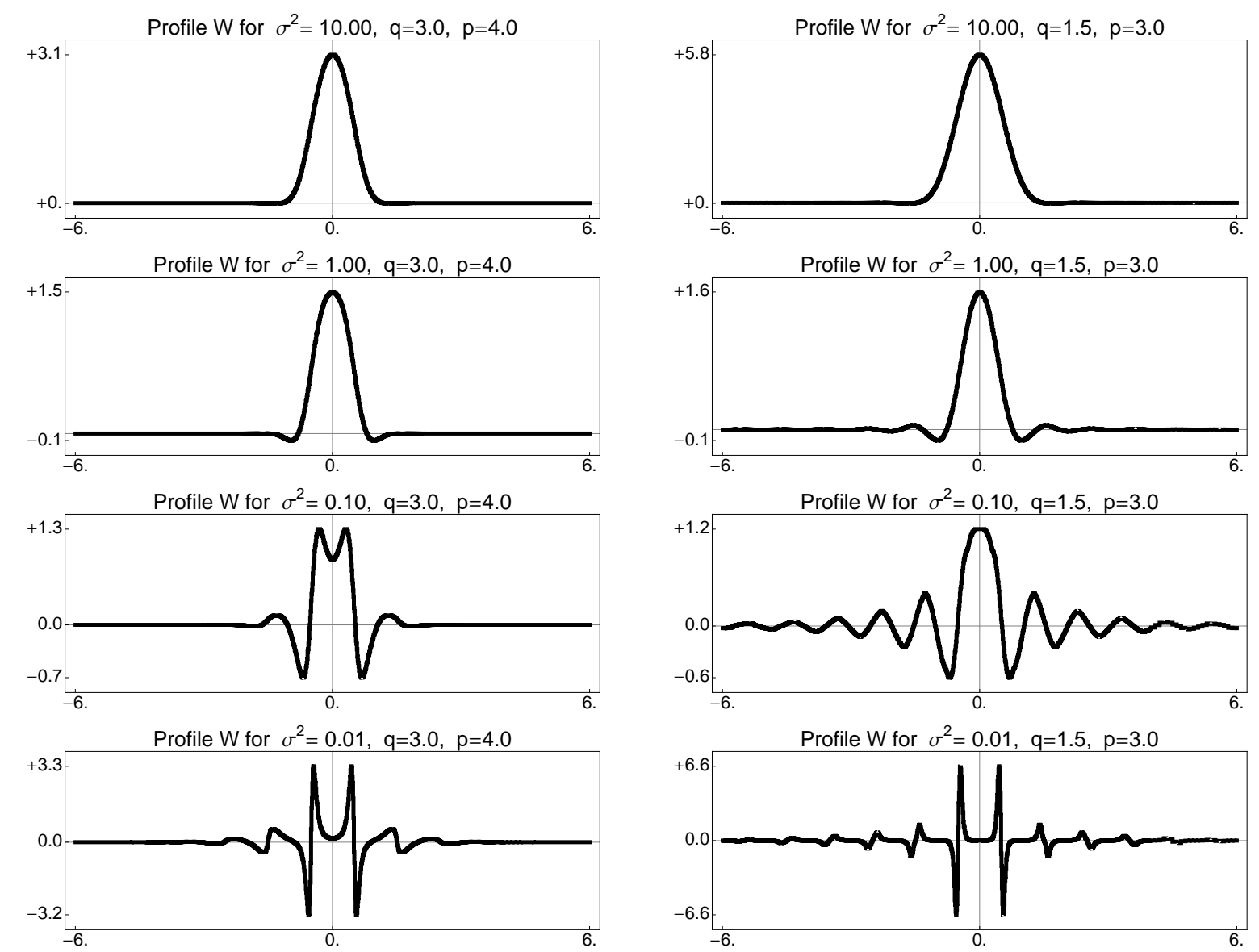

Figure 1: Numerical approximations of ground wave for different values of $\sigma^{2}$ and $q=3, p=4$ (left column) or $q=3 / 2, p=3$ (right column). The numerical parameters are $K=6, N=2400$, and $\triangle \tau \geq 0.0005$.

(b) We update in radial direction via

$$
W \mapsto\left(1+\triangle \tau \mathcal{F}_{K}(W)\right) W
$$

to enforce the constraint $\mathcal{F}_{K}(W)=0$ (notice that $\bar{\zeta}_{K}(W) \lessgtr 1$ if and only if $\mathcal{F}_{K}(W) \lessgtr$ $0)$.

3. To initialize the iteration, we discretize reasonable initial data such as $W(\varphi)=\exp \left(-\varphi^{2}\right)$.

Although this scheme is rather simple, it exhibits good convergence properties provided that the flow time $\triangle \tau$ is sufficiently small. Moreover, numerical simulations indicate that each time-discrete trajectory converges to a limit that is independent of the particular choice of the initial data, and thus we expect that this limit approximates a global minimizer of $\left.\mathcal{L}_{K}\right|_{\mathrm{M}_{K}}$.

Typical numerical solutions for $K=6$ and $\sigma \in\{0.01,0.1,1.0,10.0\}$ are displayed in Figure 1. We clearly observe that periodic ground waves are localized but have rather different shapes for large and small speeds, respectively. If $\sigma^{2}$ is sufficiently large, the double-well structure of $\Phi$ is less important and the wave looks like a unimodal wave for the convex potential $\Phi(w)=w^{p}$, see [Her10] for details. For small $\sigma^{2}$, however, the contributions from the different monomials balance and $W$ has several local extrema.

\section{References}

[FM02] G. Friesecke and K. Matthies, Atomic-scale localization of high-energy solitary waves on lattices, Physica D 171 (2002), 211-220. 
[FP99] G. Friesecke and R.L. Pego, Solitary waves on FPU lattices. I. Qualitative properties, renormalization and continuum limit, Nonlinearity 12 (1999), no. 6, 1601-1627.

[FV99] A.-M. Filip and S. Venakides, Existence and modulation of traveling waves in particle chains, Comm. Pure Appl. Math. 51 (1999), no. 6, 693-735.

[FW94] G. Friesecke and J.A.D. Wattis, Existence theorem for solitary waves on lattices, Comm. Math. Phys. 161 (1994), no. 2, 391-418.

[Her10] M. Herrmann, Unimodal wavetrains and solitons in convex Fermi-Pasta-Ulam chains, Proc. R. Soc. Edinb. Sect. A-Math. 140 (2010), no. 04, 753-785.

[HMSZ12] M. Herrman, K. Matthies, H. Schwetlick, and J. Zimmer, Subsonic phase transition waves in bistable lattice models with small spinodal region, submitted, preprint available under arXiv:1206.0268, 2012.

[HR10] M. Herrmann and J. D. M. Rademacher, Heteroclinic travelling waves in vonvex FPUtype chains, SIAM J. Math. Anal. 42 (2010), no. 4, 1483-1504.

[Ioo00] G. Iooss, Travelling waves in the Fermi-Pasta-Ulam lattice, Nonlinearity 13 (2000), 849-866.

[Pan05] A. Pankov, Traveling Waves and Periodic Oscillations in Fermi-Pasta-Ulam Lattices, Imperial College Press, London, 2005.

[PP00] A. Pankov and K. Pflüger, Traveling waves in lattice dynamical systems, Math. Meth. Appl. Sci. 23 (2000), 1223-1235.

[SW97] D. Smets and M. Willem, Solitary waves with prescribed speed on infinite lattices, J. Funct. Anal. 149 (1997), 266-275.

[SZ07] H. Schwetlick and J. Zimmer, Solitary waves for nonconvex FPU lattices, J. Nonlinear Sci. 17 (2007), no. 1, 1-12.

[SZ09] _ Existence of dynamic phase transitions in a one-dimensional lattice model with piecewise quadratic interaction potential, SIAM J. Math. Anal. 41 (2009), no. 3, $1231-1271$.

[TV05] L. Truskinovsky and A. Vainchtein, Kinetics of martensitic phase transitions: lattice model, SIAM J. Appl. Math. 66 (2005), 533-553. 